\title{
Design and Analysis of Electrical Automation Control System Based on Computer Technology
}

\author{
WANG Xiaorong*, MA Taohua \\ Inner Mongolia Technical College of Mechanics and Electrics, Inner Mongolia,010070,China
}

dqgcxwxr@163.com

\begin{abstract}
Key words: computer technology; electrical automation; control; system design
Abstract. In recent years, our country has been a great leap forward development, the level of industrialization of our country's economic and social development is to continue to improve, and this and the electrical automation control system design and application is not open. The electrical automation control system based on computer technology has the characteristics of high integration and flexible control, which is widely used in people's life. With the continuous development of social economy, the design and application of electrical automation control system is more and more people's attention. This paper mainly analyzes the design and application of electrical automation control system based on computer technology.
\end{abstract}

\section{Introduction}

Electrical automation control system is based on computer technology and electronic communication technology, the integrated control management system, electrical automation control system can achieve the optimization of the substation, not only can carry out comprehensive analysis, detection and management of the whole substation, strengthen information exchange between the various machinery and equipment, effectively realize the sharing of information resources between the machinery and equipment, strengthen the monitoring of the substation operation. In addition, the electrical automation control system has been gradually replaced by the substation two conventional mechanical equipment, simplifying the substation two times, to ensure the safe and stable operation of the substation, as far as possible to reduce the number of mechanical equipment maintenance, saving maintenance costs. Electric automation control system in industrial production, in addition to the protection and monitoring of electrical control, the electrical control of the fault in the automatic alarm, but also scientific support for the operation of power distribution system, accurate measurement of power, effective control of power consumption, real energy saving, easy for people's production and life. Thus, it is necessary to design and analyze the electrical automation control system based on computer technology.

\section{Electrical automation control system design method}

At present, the design method of the electrical automation system mainly concentrated on the way, the remote monitoring mode and the field bus monitoring mode.

\subsection{Centralized monitoring method}

The centralized monitoring system of electrical automatic control system is easy to check and maintain the operation of the system, and the design of the system is relatively simple in the protection requirements. Any centralized monitoring system must meet the needs of the system, so as to reduce the system's monitoring object and speed up the information processing speed, the function of the system must be gathered in a central processing unit. With the continuous development of science and technology, the number of electrical equipment is increasing, which leads to the increase in the number of cables, the cost increases, and even lead to the reliability of electrical automation control system. In addition, we should pay more attention to the use of hard wiring for knife gate isolation and circuit breaker interlocking operation, due to centralized monitoring mode of the system wiring is more complex, there is trouble, operation and maintenance of complex problems, therefore, in the system wiring must be more careful. 


\subsection{Remote monitoring mode}

The remote monitoring system of electrical automation control system means that the computer is connected to the computer network. Remote monitoring method can not only be controlled by the computer's desktop environment display to its own computer interface, but also to achieve the remote operation of the computer controlled, the advantages of the method is mainly to save cable material costs, low cost, high reliability, flexible configuration, etc.. In fact, most of the current communication speed can not meet the growing demand for electricity, and at some specific time also need a large amount of electrical communication, these conditions for the application and popularization of remote monitoring serious obstacles to the role of.

\subsection{Field bus monitoring mode}

In recent years, the rapid development of computer network technology based on Ethernet and field bus has been vigorously promoted and applied to the substation, which has promoted the development of electrical automation control system. With the increasing of the electrical intelligent equipment in the substation, the electrical automation control system based on computer technology is becoming an important part of the electrical system of power plant. In addition to all the advantages of remote monitoring, the field bus monitoring method has the advantages of high efficiency, saving auxiliary equipment and devices. Because of the design and installation of different devices and functions are not the same, it is only through the Internet to connect them, this way, even if one of the devices appear to be able to ensure that the other parts of the network will not affect the other parts, but will not cause the whole system to make mistakes and then crash, greatly enhance the safety and reliability of the system. In short, the research and application of field bus monitoring is very important. With the continuous progress of science and technology, the field bus monitoring mode will be fully used in the field of electrical automation control to fully play its role, and promote the electrical automation control system based on computer technology to continue to improve and develop.

\section{Design and analysis of electrical automation control system based on computer technology}

\subsection{FCS control system design}

In the electrical automation control system based on computer technology, the FCS system usually uses a pair of multi form, which is the way of transmitting signal. Because of the high accuracy of the transmission mode, the signal transmission has strong reliability, and can make the electrical equipment in the monitoring state. In the FCS control system, the problems such as lack of unified communication protocol, slow communication speed, less connection equipment and efficient combination of FCS control system, can be used in the design of FCS control system.

\subsection{Electrical load control system design}

The electrical load automatic control system is mainly used to ensure the safety and stability of the power network, the basic objective is to achieve the electrical load management and monitoring. In fact, with the continuous development of social economy, the traditional power mode to realize the management and control of the electric load has been unable to meet the people's growing demand for electricity use, this will cause the imbalance of power supply. Therefore, the traditional electric power load management is bound to develop in the direction of automation control, and gradually become an important part of the electrical control system.

At present, most of the electrical control system, can use the load system in the new electric power control system, realize the change of load control and control.

\subsection{Electrical automation communication system design}

As an important part of the electrical automation control system, the electrical automation communication system is the key to ensure the information resource transmission quickly and accurately. In fact, electrical automation control

System has a large number of terminal nodes, so the communication is very complex, the current common communication mode is the combination of wired and wireless transmission mode to achieve communication, in the choice of communication mode must be fully considered the actual situation of the electricity households, reasonable choice of the best communication. 


\subsection{Distributed control design of electrical automation control system}

Electric automation control system is the use of the central processor, intelligent instruments, network technology, inverter and low voltage circuit breaker and other organic connection, and the use of the central processing unit of the electrical equipment information to refine the processing, further to collect these data. The electric automation control system uses the distributed control method, can divide the data into the frame, it is easy for all kinds of intelligent equipment to connect the data through the automation system and the communication bus.

\subsection{Centralized monitoring and control system for electrical automation control system}

The centralized monitoring and controlling system of electrical automation control system is to focus on the information data of the electrical automation control system to the same central processing system. Because the system collects and processes the information is huge, it will slow down the speed of the system. When the electrical automation system in the detection and control of the operation, the operation of the involved more, the number of cables, occupy the host space is increased, causing the signal transmission distance becomes large, affecting transmission efficiency. Therefore, in the design of centralized monitoring of electrical automation control system, we must fully take into account the safety, accuracy and convenience of the centralized monitoring, improve the centralized monitoring design.

\section{6 remote monitoring and control system for electrical automation control system}

Electrical automation control system has the characteristics of high security, high reliability and high flexibility. It can not only save the cost, save the cable installation cost, but also reduce the cable usage and reduce the number of cable maintenance. However, electrical automation control system is difficult to establish the whole process management of electrical automatic control system, which can not meet the actual needs of the remote monitoring system. Therefore, we must take effective measures to improve the communication speed of the remote monitoring, improve the operation safety of the electrical automation control system.

\section{Electrical automation control system based on computer technology}

In recent years, the application of electrical automation control system based on computer technology is applied to all walks of life. The power plant system is analyzed, and the control system of electric power is further described.

\section{1 data acquisition and computer processing system}

Computer processing system is the basis of computer based processing system, by the input, processing, output three parts. The application of power system in power plant usually includes data acquisition, parameter input, parameter display, performance calculation, report printing, abnormal alarm, accident sequence record.

\section{2 steam turbine electro-hydraulic control system}

In the electro-hydraulic control system before China's turbine control most of the hydraulic control system, in the promotion of advanced science and technology, the reliability of electrical equipment, electrical components, electro-hydraulic converter has gradually improved. In addition, extensive application of high pressure oil servo mechanism, good coordination can realize the power transfer system and turbine auxiliary equipment, the speed, power, pressure of the governing stage control has been effectively enhanced. The steam turbine electro-hydraulic control system from the beginning to control the turning turbine wheel, and then hedge warm machine control, through the valve switch, grid, with initial load, load, power can be normal. In this process, the steam turbine electro-hydraulic control system in power grid

A frequency modulation process, through the power grid to achieve the power to change the load. Such control principle in addition to ensure the safety and reliability of the operation of the electrical units, to extend the service life of the unit, but also to ensure the stability of electrical units in the case of a comprehensive improvement in the economy. 


\section{Conclusions}

In the global economy, the role of electrical automation in China's national economy is increasingly important. The electrical automation control system has completely changed the management mode of the traditional substation, and the advanced science and electrical automation control system can ensure the safety and reliability of the power distribution and reduce the power failure. In addition, the electrical automation control system based on computer technology can not only combine the automation technology and computer technology, provide power service, but also can save energy resources, provide high quality power for people to live and produce. As a professional electrical engineering and technical personnel, must be based on the current situation of electrical automation control, to improve their overall quality, and promote the sustainable development of China's electrical automation control.

\section{Reference}

[1] Lu Haibo. Design of electrical automation control system [J]. Electronic technology and software engineering.2015 (02): 170-171.

[2] Xu Wenbin. Design idea of medium-sized enterprises and technology electrical automation control system[J]. Management and technology , 2012 (03): 284-285.

[3] Xin Xin, Zhou Rui frost. The use and development of the electro pneumatic automatic control system [J]. Heilongjiang science, 2014 (04): 81-82.

[4] Tang Yifan. Electrical automation control system design under the application of computer technology[J]. Electronic test. 2014 (10): 97-98+96.

[5] Li Hui, Wang Haifeng. Analysis of the design of the electrical automation control system [J]. Henan technology, 2013 (02): 100-101.

[6] Zhang Xiaobo, Zhang Yan. Design and application of electrical automation control system based on computer technology [J]. Electrical and mechanical information, 2012 (15): 144-145. 